\title{
REFLEXIÓN SOBRE LAS CLASES DE LENGUA ESPAÑOLA DURANTE LAS PRÁCTICAS DE FORMACIÓN DOCENTE
}

\author{
REFLECTION ON SPANISH LANGUAGE CLASSES \\ DURING TEACHING TRAINING PRACTICES
}

\author{
Daniela Moro ${ }^{1}$ \\ Isaphi Marlene Jardim Alvarez ${ }^{2}$
}

\section{PRESENTACIÓN}

Este relato de experiencia pretende reflexionar sobre algunos aspectos que hacen parte del cotidiano de un profesor de lenguas. De ese modo, como graduanda del curso de Letras - Línguas Adicionais: Inglês, Espanhol e Respectivas Literaturas de la Universidad Federal do Pampa (UNIPAMPA), establezco algunas cuestiones relacionadas a las diferentes metodologías, objetivos y perfiles de la enseñanza de lengua española, considerando aspectos contextuales que involucran estas acciones. Sobre las clases impartidas en la práctica, cabe aclarar que incertidumbres sobre como ministrar clases de lengua, y de modo más específico, de lengua española, también son sentidas por los docentes de la enseñanza básica en las escuelas. Hay, por lo tanto, la necesidad de una reflexión mayor acerca de la formación del profesional de lenguas que actúa en la educación básica brasileña.

Las clases oriundas de las prácticas ofrecidas por nuestro curso permiten que los estudiantes reflexionen sobre las metodologías y estrategias de enseñanza en el proceso de aprendizaje de lenguas dentro de las escuelas, de modo a considerar la comunidad y el contexto social del profesor y de los alumnos en el momento de la práctica. Por consiguiente, el objetivo de este trabajo es reflexionar sobre los desafíos encontrados en la enseñanza de español como lengua adicional ${ }^{3}$ en $\mathrm{mi}$ formación docente. El elemento motivador está directamente relacionado a las oportunidades concedidas en las prácticas, comprendidas como una forma de pensar en las problemáticas de la enseñanza pública y de añadir nuevas experiencias y nuevos puntos de vista al aspecto de la producción de materiales para clases futuras. La construcción de los saberes necesarios para la práctica pedagógica es una tarea compleja y exige del profesor un amplio enfoque en sus primeras etapas de formación y de experiencia en el profesorado.

\section{CARACTERIZACIÓN DE LA ESCUELA}

Las prácticas en lengua española se llevaron a cabo en una escuela pública, situada en el centro de la ciudad de Bagé, en Rio Grande do Sul. Fueron impartidas 18 clases para dos grupos de segundo año de la secundaria, con un total de 34 (treinta y cuatro) alumnos, 17 (diecisiete) en cada grupo, en el período nocturno. Además, tuve la posibilidad de observar 2 clases antes de empezar mis prácticas, con el objetivo de conocer y establecer los perfiles y el contexto de la enseñanza de español en la escuela.

\footnotetext{
${ }^{1}$ Graduanda do curso de Letras: Línguas Adicionais: Inglês, Espanhol e Respectivas Literaturas da Universidade Federal do Pampa. Contato: danielasteffenmoro@gmail.com.

2 Professora doutora da Universidade Federal do Pampa. Contato: isaphi.jalvarez@gmail.com.

${ }^{3}$ Usamos el término lengua adicional (LA) porque entendemos que el concepto permite una visión más amplia y completa, ya que considera la nueva lengua como una adición, puesto que todos ya tenemos al menos una lengua, nuestra lengua materna. Para más informaciones, leer Leffa e Irala (2014). Disponible en: http://www.leffa.pro.br/ textos/trabalhos/livro_espiadinha.pdf. Acceso en: 30 mayo. 2019.
} 
La profesora titular hizo su graduación en UNIPAMPA e imparte clases en la ciudad hace diez años, en escuelas públicas de la red municipal y departamental o de la provincia. Por esa razón, compartimos metodologías y abordajes de enseñanza con fundamentaciones semejantes y pudimos establecer algunos conceptos y perspectivas comunes, intercambiando experiencias y nuevas ideas para la elaboración de mis primeras clases. No obstante, tuve muy poco contacto con los demás profesionales de la escuela, pues estaban siempre demasiado ocupados con sus tareas, comúnmente divididas en más de una institución.

Esa condición se impone y no podemos dejar de mencionarla, debido a las inúmeras dificultades que estos profesionales viven en las escuelas públicas, sea por los bajos sueldos, sea por la falta de infraestructura, ya que, en ámbito general, se les ofrece una labor cotidiana con muchas horas de trabajo, con muchos grupos para atender y con una lamentable valoración de su trabajo como docente, pues sabemos que cada vez se invierte menos en educación en Brasil.

Los grupos tenían alumnos con edades muy variadas, entre 15 (quince) y 40 (cuarenta) años. Los estudiantes, en su mayoría, trabajaban durante el día y estudiaban por la noche. Poseían un nivel de conocimiento muy básico del español, aunque ya habían estudiado la lengua en años anteriores. Ellos también poseían niveles de adquisición lingüística diferentes, ya que algunos nunca habían tenido contacto con la lengua española en la escuela y otros habían vivido en ciudades como Rivera, Aceguá y Buenos Aires, o tenían contacto con la lengua por medio de otras personas, como sus abuelos o amigos hispanohablantes ${ }^{4}$.

A continuidad, a través de las observaciones pude percibir las distintas posibilidades y contextos de enseñanza que los futuros profesores de lengua pueden encontrar en la caminada docente. Así pues, la dedicación del profesional frente a los alumnos, sumado al planeamiento y a la producción de materiales didácticos, es uno de los primeros pasos para alcanzar el futuro éxito escolar. Por lo tanto, pensar en tareas que presenten a estos alumnos modos críticos de observar a la sociedad y de construir sus identidades debe constituir toda la trayectoria del profesional de lenguas

\section{FUNDAMENTACIÓN TEÓRICA}

Para la realización de esta investigación, utilicé el PNLD (2017), que destaca el uso de la lengua como modo importante de aprenderla y el guion, creado por el Ministério da Educação (MEC), que también aborda cuestiones relacionadas a la enseñanza del idioma y a la selección y elaboración de materiales didácticos. Como aporte metodológico de proyecto de práctica, recurrimos a LATORRE (2003) que plantea la investigación como "una forma de indagación realizada por el profesorado para mejorar sus acciones docentes o profesionales y que les posibilita revisar su práctica a la luz de evidencias obtenidas de los datos y del juicio crítico de otras personas" (LATORRE, 2003, p.5). En esa dirección de reflexión, LEFFA e IRALA (2012) aportan discusiones a respecto de la enseñanza de lenguas en la contemporaneidad, afirmando que más allá de la pretensa enseñanza comunicativa, hay resquicios de conceptos considerados superados, como la concepción de que enseñar lengua es enseñar gramática, muy presente en las escuelas. Así pues, el presente trabajo muestra el abordaje y la organización de las clases de practica a través del uso de géneros textuales (MARCUSCHI, 2013), que circulan en diferentes ámbitos sociales.

\section{DESCRIPCIÓN DE LA EXPERIENCIA}

El proyecto tuvo como primer objetivo empezar las prácticas en lengua española en la asignatura de Estágio em Contexto Escolar I, a partir de un enfoque comunicativo, pese a conocer el ambiente escolar, sus desafíos y posibilidades. Por un lado, decidí trabajar con contenidos que podrían ofrecer a los alumnos de la escuela la oportunidad de desarrollar pensamientos críticos y

\footnotetext{
${ }^{4}$ Puede ser común encontrar en las escuelas de Bagé estudiantes que tengan contacto con hispanohablantes o una relación más cercana con la lengua, debido al contexto fronterizo de la ciudad con el vecino país Uruguay.
} 
significativos frente a los contextos sociales vivenciados por ellos. Por otro lado, los alumnos pudieron posicionarse, indagarse y modificarse frente a las diferentes perspectivas y puntos de vista en relación con su comunidad.

Todas las clases fueron elaboradas para que hubiera un espacio de conversación sobre temáticas que abordaban problemas pertinentes a la sociedad. Igualmente, las clases estaban centradas en aspectos lingüísticos que podrían ayudar a los alumnos a desarrollar el pensamiento crítico (LATORRE, 2003) por medio de la lengua española.

Primero, los alumnos fueron presentados a dos diferentes géneros discursivos: la historieta ${ }^{5}$ y el fanfic ${ }^{6}$. A partir de los textos y aspectos lingüísticos presentados, al final de la práctica produjeron sus propios fanfics, relacionándolos con las temáticas sociales más relevantes para cada uno, de modo colaborativo.

En efecto, la escuela no poseía un amplio aporte de elementos tecnológicos, tampoco los estudiantes tenían acceso a internet. Por tanto, los materiales utilizados en las clases fueron adaptados para hojas guiadas con las definiciones de los diferentes géneros textuales, previamente discutidos, así como los contenidos gramaticales propuestos como apoyo para las producciones. Conviene resaltar que la falta de acceso a las tecnologías, a pesar de ser una desventaja para la escuela, no perjudicó el desarrollo de las clases y tampoco la participación de los estudiantes en las tareas propuestas.

Los alumnos recibieron algunas de las historietas de Mafalda ${ }^{7}$ (Apéndice 1 y 2) para, a partir de ellas, instigar su pensamiento crítico acerca de las temáticas sociales subyacentes. Posteriormente a la comprensión de las historietas, los alumnos fueron introducidos al género fanfic, además de tener contacto con el subgénero Crossover. También pudieron hacer investigaciones acerca de las historias producidas en los distintos géneros y compartir en las clases lo que descubrieron. Los alumnos trabajaron con la producción de brainstormings ${ }^{9}$ (Apéndice 3 y 4), con el objeto de comprenderlo como una posibilidad de uso para la producción inicial de textos y para el levantamiento y discusión de ideas. Ellos también realizaron tareas sobre las conjugaciones de verbos en el presente de indicativo y aprendieron nuevos vocabularios. Estos aspectos lingüísticos, aunque no fueron profundizados en esta práctica, se han convertido fundamentales para la comprensión y elaboración de los géneros trabajados.

En sus producciones, los alumnos desarrollaron, en parejas o en grupos, un fanfic que debía involucrar una de las temáticas que más les llamó la atención durante las clases. A partir del tema, tuvieron la oportunidad de desarrollar una reflexión crítica, colectiva, por medio de discusiones sobre asuntos que eligieron como importantes desde su realidad. Las producciones podrían ser en forma de texto corto o historieta (Apéndices 5, 6 y 7). Todos los alumnos eligieron la producción en forma de texto corto porque se sentían más confortables para escribir solamente los textos, sin la necesidad de hacer dibujos o preocuparse con la parte estética, aunque esto les exigía un desarrollo mayor en la producción escrita.

Los estudiantes también pudieron trabajar con sus personajes favoritos, trayéndolos de las obras de ficción que más les gustaba y contextualizándolos en las diferentes temáticas. Si estaban

\footnotetext{
${ }^{5}$ Comprendemos historieta como la narración de una historia a través de una sucesión de ilustraciones que pueden complementarse con un texto escrito y que generalmente cumple la finalidad de hacer una crítica social.

${ }^{6}$ Comprendemos fanfics como relatos de ficción escritos por fans de alguna obra literaria, que añaden nuevas circunstancias para desarrollar la historia más allá de la original. Las historias tienen la intención de entretener o pasar alguna lección.

${ }^{7}$ Nombre de una tira de prensa argentina que fue desarrollada por el humorista gráfico Joaquín Salvador Lavado Tejón, mejor conocido como Quino, en las décadas de 60 y 70, que tenía como personaje principal una niña homónima, de la clase media progresista de Argentina, que tiene una preocupación sagaz con la humanidad y critica el mundo construido por sus mayores.

${ }^{8}$ Comprendemos crossovers como historias que mezclan dos o más series distintas en que personajes de diferentes obras interactúan entre sí.

${ }^{9}$ Comprendemos brainstorming como una técnica de creatividad, realizada en grupos o individualmente, que tiene como objetivo principal exponer las ideas acerca de un determinado tema, o mejorar las resoluciones ya existentes.
} 
trabajando en parejas, deberían hacer su personaje favorito interactuar con el de su compañero en el tema que eligieron juntos. La elección de los personajes ocurrió como medio de hacer la escrita del fanfic atractiva e interesante a los alumnos, de modo que podrían utilizarlos como apoyo para intentar resolver problemas sociales muchas veces complejos y difíciles. Así, la lengua española se hizo pertinente como una herramienta para la producción escrita que buscaba contemplar la realidad de aquellas personas.

En el último día de práctica, los alumnos presentaron, en lengua española, sus producciones para los compañeros. Los fanfics, en general, permitieron a los estudiantes reflexionar críticamente y colaborar para la búsqueda de soluciones. Los textos de los alumnos estaban muchas veces cargados de emociones y presentaban la realidad de sus vidas, con temáticas que iban desde los malos tratos a los animales y la virtud de la humildad, hasta asuntos más complejos como el racismo, el uso de drogas y la corrupción. Todos tuvieron la oportunidad de agregar opiniones y sugerencias a los tópicos presentados por los compañeros y así ayudar a construir los trabajos colaborativamente.

\section{METODOLOGÍA}

La metodología utilizada en este trabajo tuvo por base la investigación-acción, que consiste en una serie de actividades que vislumbran contribuir con el sistema educativo. Según Latorre (2003), la investigación-acción se utiliza para describir un grupo de actividades que los profesores realizan en sus clases con fines de desarrollar aspectos curriculares, profesionales, mejorar programas educativos, sistemas de planificación o la política de desarrollo.

La justificativa de la elección de esta metodología es justamente la importancia de la reflexión acerca de los desafíos encontrados en la enseñanza del español como lengua adicional, teniendo en cuenta la enorme responsabilidad de los docentes de lengua, ya que se trata del profesional que participa de la formación ciudadana de las personas que constituyen la sociedad.

\section{ANÁLISIS DE LOS RESULTADOS}

Como la lengua está relacionada con las experiencias de interacción presentes en el convivio social, su uso debe considerar una gran variedad de géneros del discurso y sus diferentes posibilidades. Estas interacciones muestran la diversidad de textos que circulan socialmente (MARCUSCHI, 2003). Es decir, saber una lengua adicional es también saber utilizarla en diferentes usos del lenguaje, marcados por un contexto social específico.

En mi práctica, los alumnos tuvieron contacto con las historietas de Mafalda, con el objetivo de reflexionar sobre los problemas sociales existentes en diversos contextos sociales, incluso los de ellos mismos. Además, tuvieron la oportunidad de conocer los géneros fanfic y crossover y producir por primera vez su propio texto en lengua española. En mis clases, la concepción de género textual siguió la de MARCUSCHI (2003), en que se refiere a los vocablos como:

[...] textos materializados que encontramos en nuestra vida diaria y que presentan características socio-comunicativas definidas por contenidos, propiedades funcionales, estilo y composición característica" (MARCUSCHI, 2013, p. 4).

Uno de los desafíos más importantes para un estudiante y futuro profesor de lenguas es saber cómo poner en marcha las teorías aprendidas. Por lo tanto, la práctica permite a los profesores en formación experimentar las clases de lenguas y proporcionar una vivencia anticipada de la profesión. Conforme con Leffa e Irala (2014), "no hay una necesidad de discriminar el contexto del otro, sino una construcción de aspectos sistémicos, de práctica social y construcción de sujeto" (LEFFA; IRALA, 2012, pg.32).

En lo que se refiere a la enseñanza de español como lengua adicional, las clases pueden ofrecer condiciones para que los estudiantes busquen comprometerse en actividades en las cuales el

https://periodicos.unifap.br/index.php/letras

Macapá, v. 9, n. 4, $2^{\circ}$ sem., 2019 
uso de la lengua-objeto proporcione reflexiones sobre temáticas importantes al contexto del alumno y dirija su atención a la comunidad por medio de la comprensión sobre el local en que vive.

Por eso, al comenzar mis clases de lengua española en la escuela, busqué primeramente conocer mejor a los alumnos y el contexto a que ellos pertenecen. A partir de las informaciones obtenidas, tanto en la observación de las clases impartidas por la profesora titular como durante mis primeros contactos con los alumnos durante la práctica, empecé a pensar y elaborar las clases de lengua dirigidas a la reflexión sobre esos importantes aspectos.

Así, trabajé el español con el propósito de desarrollar el pensamiento crítico, a partir de una pedagogía dialógica que, conforme LEFFA e IRALA (2014), permite al profesor construir, en conjunto con el alumno, el conocimiento lingüístico que valora el propio contexto en que ambos viven. En consecuencia, los alumnos tuvieron la oportunidad de traer a la clase las temáticas que consideraban pertinentes para la realidad social en que están insertados y que forma parte de sus concepciones como ciudadanos.

La comunidad escolar también parecía tener una resistencia en relación con el uso del español en las clases. Antes de empezar mi práctica, tanto los alumnos como la profesora no hablaban español. Los estudiantes rechazaban el idioma y, con frecuencia, se utilizaban como ejemplo al mencionar que la propia lengua portuguesa les parecía muy difícil. Creían que no eran capaces de adquirir otro idioma, aunque tenían el deseo de aprender. Al reflexionar y ampliar mis perspectivas sobre tales aspectos, me pareció pertinente desarrollar una metodología en la que los alumnos se sintieran confortables durante el uso de la habilidad oral en clase. Igualmente, busqué enfocar primariamente el uso de la lengua española en tareas de producción escrita. Por medio de la lengua en uso (PNLD, 2017), los alumnos pudieron empezar a desarrollar el idioma con el fin de comunicarse, siempre considerando su propio contexto y conocimiento de mundo. Así, las cuestiones referentes a los aspectos gramaticales que surgieron entre los estudiantes eran discutidas en clase a partir de las propias producciones.

Estos desafíos también se repitieron en las clases de algunos compañeros del componente curricular, puesto que comúnmente compartían los mismos relatos en lo que se refiere a los miedos y anhelos de sus alumnos frente el aprendizaje de nuevas lenguas. Estos aspectos reiteran la importancia de ampliar la reflexión sobre ellos, de modo a poner a disposición del alumno la familiarización y la sensibilización para el aprendizaje de lengua.

\section{CONSIDERACIONES FINALES}

A partir de lo que fue presentado en este relato y de acuerdo con los teóricos referidos, concluyo que las prácticas de lengua española me conectaron con la realidad de los alumnos y la vivencia diaria de la docencia. Hay muchas dificultades encontradas en este proceso de enseñanza y aprendizaje. Sin embargo, es necesario mantener la constante búsqueda por metodologías y estrategias de enseñanza que atiendan a las demandas de los alumnos y a las del sistema educacional.

La realidad de las escuelas muchas veces exige innovaciones en las prácticas pedagógicas. Usando la lengua adicional como una importante herramienta de comunicación, es posible llevar a los estudiantes cuestiones sociales y culturales, de manera que sus discusiones posibiliten el acceso a diferentes perspectivas y al desarrollo del pensamiento crítico de los alumnos.

Así pues, otros estudios pueden ser desarrollados con el fin de buscar el perfeccionamiento y la superación constante de los obstáculos de la enseñanza de la lengua española en el sistema de enseñanza pública, con el fin de atender a las demandas de los alumnos y ciudadanos que constituyen nuestra sociedad. 


\section{REFERENCIAS}

LATORRE. A. La investigación- acción. Conocer y cambiar la práctica educativa. Barcelona: Graó, 2003.

LEFFA, V; IRALA, V. B. O ensino de outra(s) língua(s) na contemporaneidade: questões conceituais e metodológicas. In : L. V.; IRALA, V. (Orgs.). Uma espiadinha na sala de aula: ensinando línguas adicionais no Brasil. 1 ed. Pelotas: EDUCAT, 2014, p. 21-48.

MARCUSCHI, L. A. Gêneros Textuais: definição e funcionalidade. In: DIONÍSIO, Â. P.; MACHADO, A. R.; BEZERRA, M. A. (Orgs.). Gêneros Textuais e Ensino. 2a ed. Rio de Janeiro: Lucerna, 2003.

PNLD. 2017. Lingua Estrangeira Moderna. Disponible en: http://www.fnde.gov.br/pnld-2017/>. Acceso en: 16 jun. 2018.

Recebido em: 23/01/2019

Aprovado em: 12/04/2019

\section{Apéndices 1 y 2}

Ejemplos de las historietas de Mafalda trabajadas en clase.
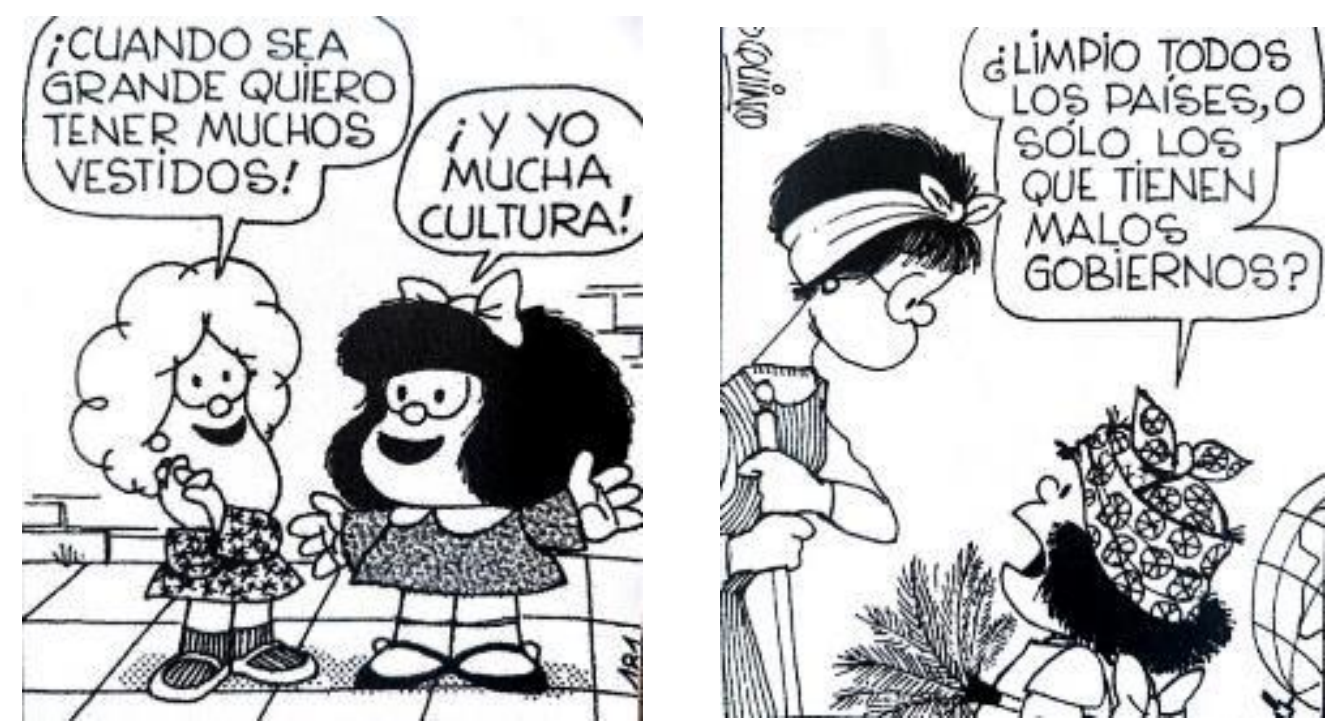

Fuente: SANTOS, A. Espanhol sem Fronteiras. 2016. Disponible en: https:// espanholsemfronteiras.com.br/mafaldatirinhas-e-historia/. Acceso en: 17 jun. 2018. 


\section{Apéndices 3 y 4}

Primera producción del brainstorming.

¿Qué es un Fanfle?

Un fanfiction es la ficción sobre la ficción.

Es la imaginación llevada al extremo; es ese paraíso en el que ocurre todo aquello que no sucede en la obra original. Un fanfic está lleno de locuras, amores prohibidos, escenas eliminadas, historias del día a día, argumentos revueltos y personajes en nuevos entornos.

Para que os hagáis una idea, las cifras que maneja FanFiction.net (la web más popular en el medio) rondan el millón de fanfics escritos para la categoría de libros, estando en cabeza Harry Potter, con 728.ooo fanfics. En el caso de los videojuegos nos encontramos con más de 500.00o relatos, estando en el número uno Pokémon, con casi 84.000. Sin embargo, en estas cifras no se incluyen aquellas historias del subgénero Crossovers. Un crossover es simplemente la interrelación de historias o personajes de diferentes lugares. Un ejemplo puede ser la película Alien vs. Depredador.

Dado que la mayoría de los fies están motivados por relaciones entre personajes, que normalmente no ocurren en la versión original, el fanfic es la escapatoria perfecta para imaginar nuevas situaciones y finales. Fs un lugar de creación donde todas las fantasías que puedas imaginar ocurren gracias al maravilloso talento de aquellos que consiguen hacer realidad lo que no pudo ser en la historia original.

Texto adaptado de: https://medium.com/espanol/qu\%C3\%A9-es-un-fanfiction-a9942d6321ae

\section{Brainstorming:}

iAhora es tu turno de pensar en la creación de un Fanfic! A continuación, escribe el personaje que más te gusta y escribe el problema social que más te molesta, pensando en cómo él podría ayudar a solucionarlo. iPiensa también en la posibilidad de la participación de Mafalda en el mismo universo! Escribe también palabras que tú consideras importante para la creación del Fanfic.

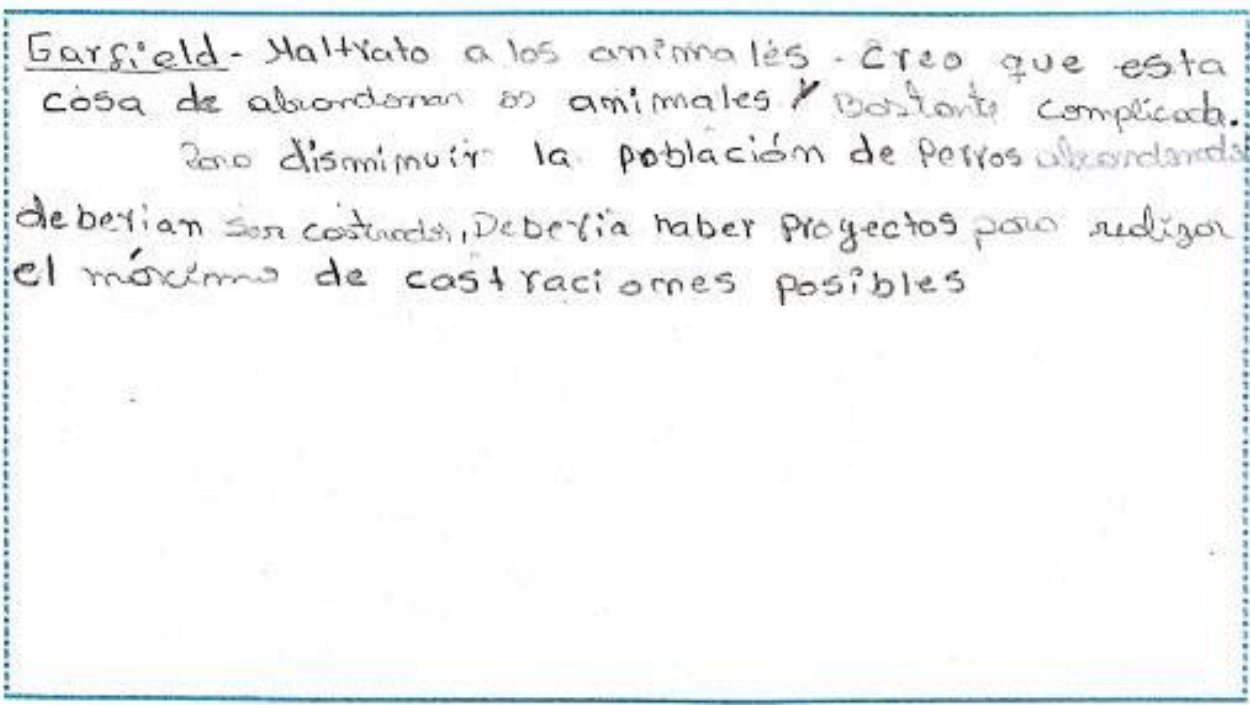

Fuente: La Autora (2018) 


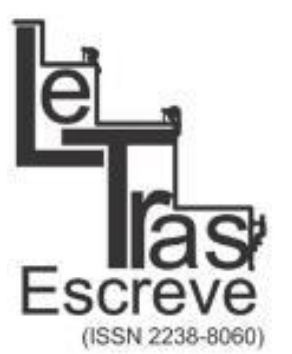

Imagen 4: Producción de lluvia de palabras después del brainstorming hecho colectivamente.

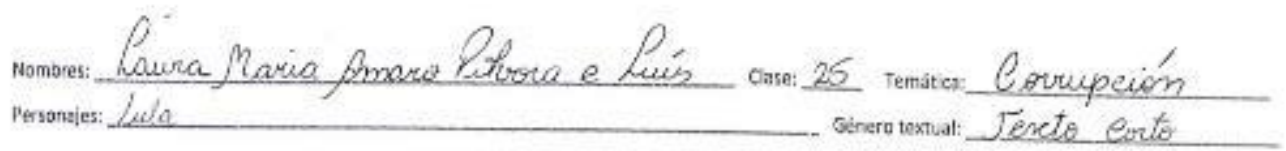

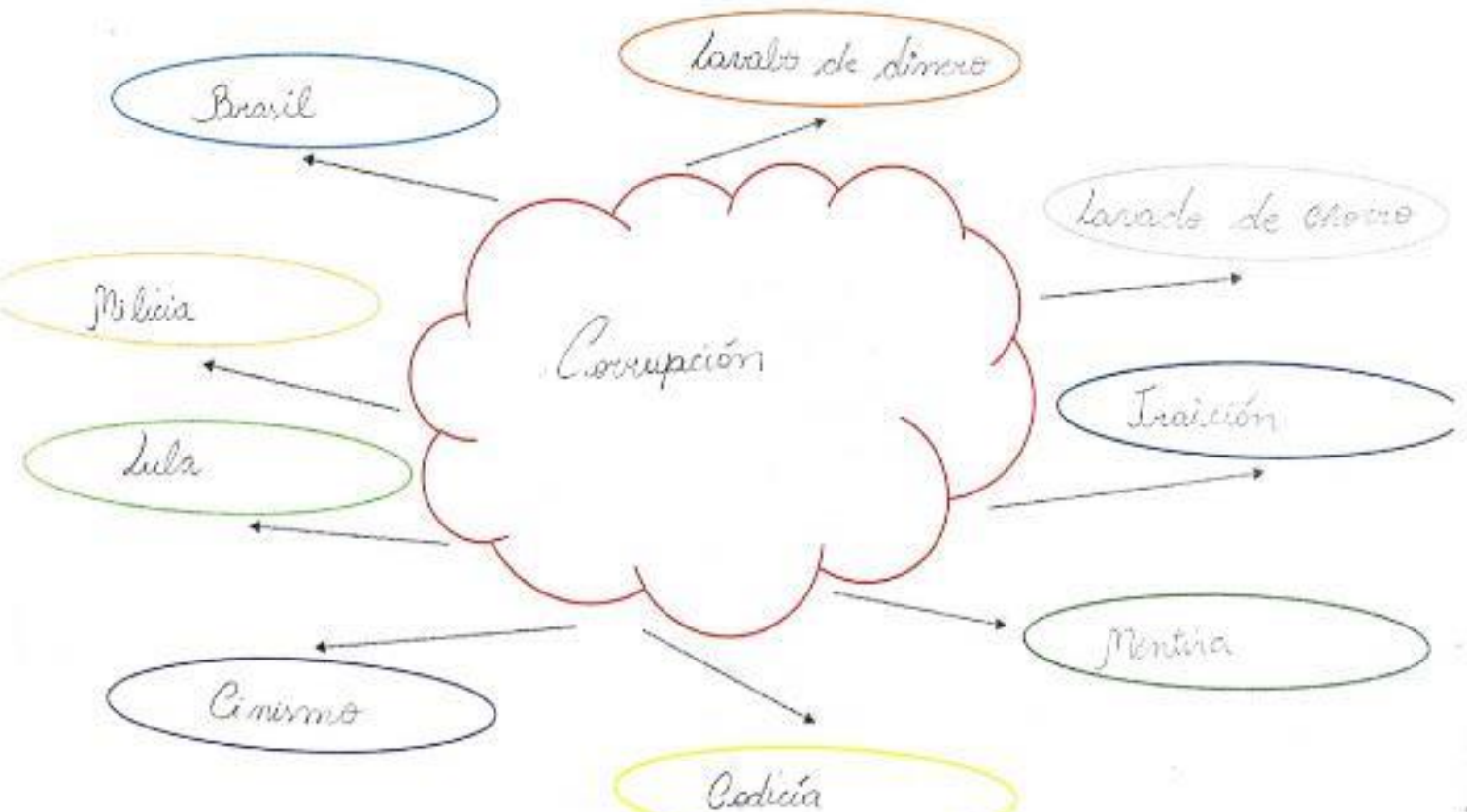

Fuente: La autora (2018).

https://periodicos.unifap.br/index.php/letras

Macapá, v. 9, n. 4, $2^{\circ}$ sem., 2019 


\section{Apéndices 5, 6 y 7}

Ejemplo de producción del texto corto.

Titulo: Covrupción no Brasil

Era epoca de elección y el persomal estala an cianor para zer quién sría el muerio alcade fe la cuidad, embue los competi dores estriba. Leomarde prequito, un señer cle 40 años que arates de presentaris a alcade era propictario de una plantación cle quayala de dar emvidio. Era un hombre raptroso perer que en el fundo temía buerax inten ciones. Leorardo ougusto us postuló con la intonción cle clar al pueblo lo que

munca dierom, mejones. del asunte y dicía que cuando fuera a vetar optaria por amular, porqué para Chico Bento todos los candidatos era sirmpre la misma cosn, pres. - meter, si elegie y nor cumprir com rada. Esta urg me fue diferente, Leomardo Angusto fue elegido, y con el demero del pueter cumplís com gegunas de sus fromesas e hizo algumas reformas en la ciudad, pere com Llesto él hizo caja dos. y come de lo lrueno y lo mejor y sus 2 rotants aque comfiarom on sus palabras estom con salárices lojiss y impuestos más altos no consiguiendo usestener a sus famílias $x$ casas.

Chice Bento, viende esa situación, refela: "A cada cuatro años es lo mismo cesa, la Gemte habla que ticme que acalrar com esso corrupción Y .ter minamos votando en los que. más roln? \& Ronqué lo hacen? Ror qué en su salegar ellos "noban perr hacin".

Moraleja de la historia:

Quién mecerita cambrior somos mesotros los votantes, muestras opiniones, perque mien-

tras pensamos de esa manera, va a encis- Tir é cerrupción. 


\section{Texto corto}

Titulo: 5 COOBY-DO EN MALTRATO A LOS ANVMARALS 5 coole - do viváa con sue prom omigo salsicha que necusitala hocen un viajey lo dejió, con sus tíos. Gus tios de Salcichas

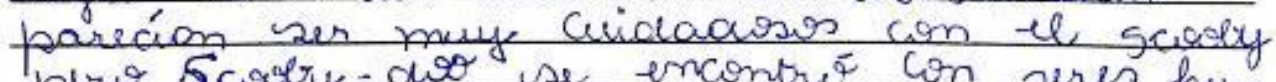
pero Scodry-doo se encontro con seres her chonos cruveles.

- Scooery precieró que los seres humsonos pue. den ser muy marredos pare un animal inav. fense, pues su gron omigo lo onole como hodie, perque los itres persinas nu w omalon, inclusio eles me mbitratonow, los ono. Reflención: Porque si alimentames a une
persiona por treinla onos, el se ifidaré de ti en tres dios. 
Texto corto

Titulo:

lick istá plamendes dag pora ocolor com da

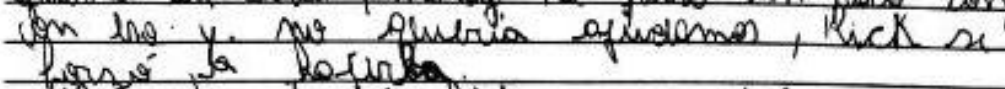

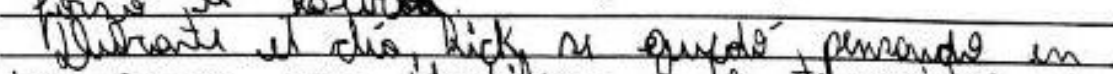

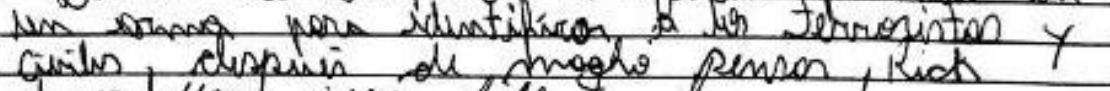

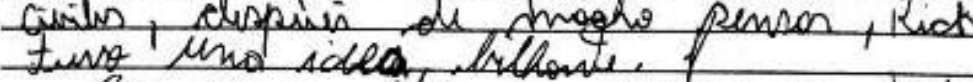
Su erana itra a succiones tedo el omomente olit nas hick $n$ elesploge- for los dimensiones pera forar los tetnotopios esencieds

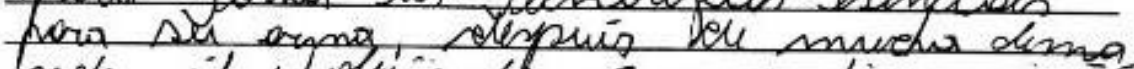
molu il. Voliviend a sel dimmonien rick commat a pensen cuéne serwón si orma, di inchura del peso y la evructura coll ella, in el gavie

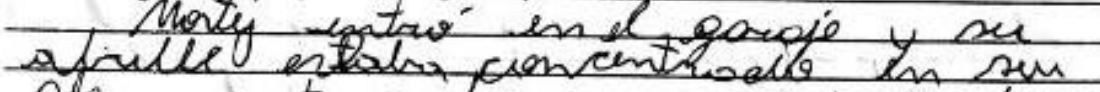

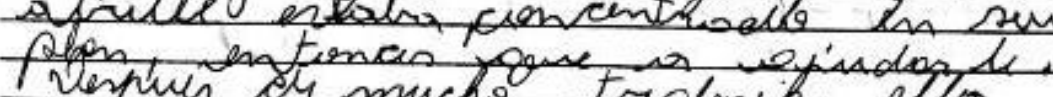
vespun \&4 mucho tradrapte eltos Heperwen in pentichs cencrien an el prona sitra a ces unsa. aspula for lemp fodi Siries y Hacis oncitiof

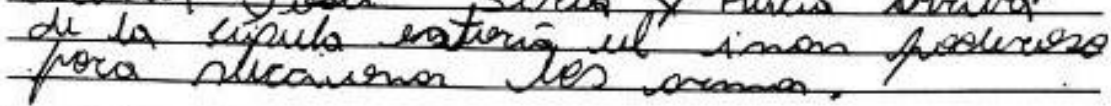

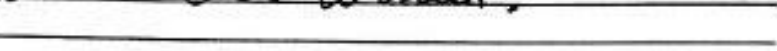
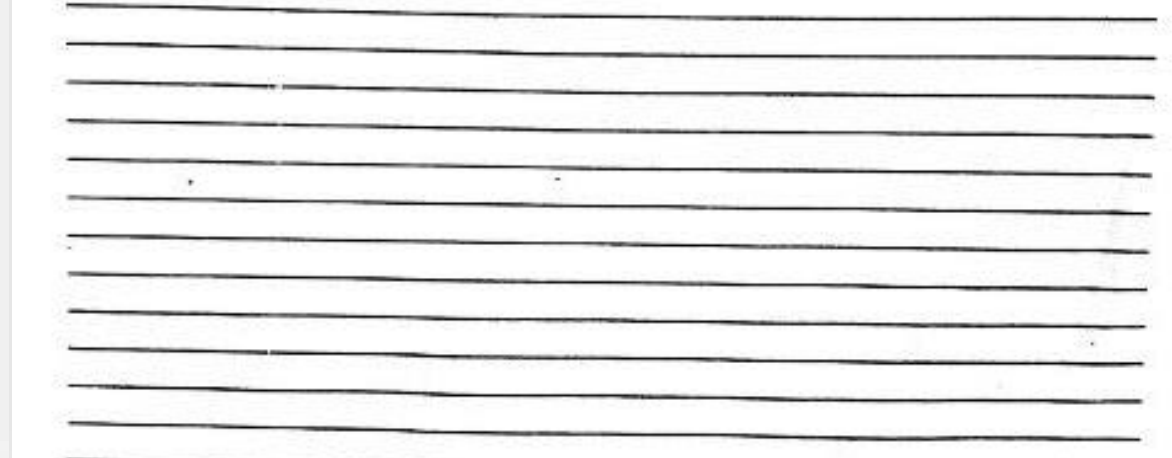